\title{
Soutien des équipes de santé mentale aux équipes MCO : une expérience de la crise Covid
}

\section{Mental health care's team support to MCO teams: A Covid crisis experience}

\author{
Ivan Ehmke ${ }^{1}$, Marie-Françoise Charpin ${ }^{1}$, Florent Girin ${ }^{1}$, Laurent Hersent ${ }^{1}$, Isabelle Odin ${ }^{1}$ \\ et Magali Briane ${ }^{1}$ \\ ${ }^{1}$ Clinique Mon Repos, Ramsay Santé, France
}

\begin{abstract}
Résumé. Nous avons développé depuis plusieurs mois une expertise sur la prise en charge des soignants, grâce à un pôle de soin spécifique. Le modèle thérapeutique utilisé s'appuie sur les thérapies comportementales et cognitives et sur la thérapie d'acceptation et d'engagement (ACT). Nous avons été sollicités début avril 2020 dans le contexte de la crise Covid, pour accompagner les équipes d'une clinique MCO (Médecine - Chirurgie - Obstétrique) de la région Parisienne. Le contexte d'intervention a permis d'identifier des critères d'alerte vis-à-vis de l'urgence d'une réponse face à la crise sanitaire. Par la suite, des besoins ont été identifiés à travers divers partenaires. La prise en charge s'est vue nécessairement en groupe et la qualité des intervenants a été une des clés du dispositif. Celui-ci s'est déployé dans trois services : un de médecine, un de réanimation et un d'urgence. Plus spécifiquement, les outils de la thérapie ACT, à différents niveaux, viennent explorer les ressources et favoriser un engagement vers le changement, tout en accueillant la difficulté de l'entreprendre face à l'arrêt d'un processus de penser. Le sens, de manière générale, s'est établi à travers la verbalisation et le débriefing, permettant un fil conducteur pour les équipes. Ainsi, en abordant d'autres perspectives, la temporalité de crise se voit transformer, et dépasse un certain désespoir vécu.
\end{abstract}

\begin{abstract}
For many months, we have been developing a specific care of healthcare workers thanks to a new department. We were using behavioral and cognitive therapy, and acceptance and commitment therapy (ACT). We have been solicited in April 2020 during the Covid health crisis to support teams working in $\mathrm{MCO}$ in the Parisian region. Alert criterions have been identified in regards of the Covid health crisis. In order to apprehend the team's needs, several exchanges with specific partners have been carefully monitored. It appears that working with a group of person was more beneficial rather than one at a time. Also, the use of a pair of mental health worker has been a key point in the whole process. It has been done in three departments: medicine, intensive care, emergency. The tools of the ACT, at different levels, allowed to explore resources and to favor commitment toward valued behavior, while welcoming the trouble of undertaking it. One of the restraint has been the stopping of a thinking process. The meaning, as a whole, has been found thanks to verbalization and debriefing ways, allowing a common thread for all the teams. Thus, by exploring new perspectives, the temporality of the crisis can be altered, overtaking despair.
\end{abstract}




\section{Présentation du contexte d'intervention}

Le centre de jour de la clinique mon repos à Ecully a développé depuis plusieurs mois une expertise sur la prise en charge des soignants et personnels médico sociaux ainsi qu'un pôle de soin spécifique pour ces personnels. Le modèle thérapeutique proposé s'appuie sur les Thérapies Comportementales et Cognitives et plus particulièrement sur la Thérapie d'Acceptation et d'Engagement (ACT). Ce modèle est issu des travaux de Steven C. Hayes (Hayes, 2004). Forte de cette expérience, l'équipe médico-soignante du Centre de Jour a été sollicitée début avril 2020 dans le contexte de la crise Covid, pour accompagner les équipes d'une clinique MCO de la région Parisienne.

Comme nombre d'établissements de santé, pendant la crise sanitaire, l'activité de cette clinique MCO a été bouleversée. L'activité MCO programmée a été annulée sur demande des ARS (Agence Régionale de Santé), pour permettre, dans le cadre du Plan Blanc, la collaboration souhaitée entre secteur public et privé. L'établissement demandeur situé en zone où la crise sanitaire était particulièrement intense a donc accueilli de nombreux patients positifs au Covid.

Ainsi, un service de chirurgie ambulatoire est devenu un service de médecine Covid, des lits de réanimation Covid ont été armés au bloc opératoire et les urgences ont été réorganisées avec une filière urgences classiques et urgences Covid. L'établissement a accompagné les montées en compétence du personnel. Les équipes de soins qui ont été mobilisées, pour une part sur la base du volontariat, ont dû faire preuve d'une grande adaptabilité.

Plusieurs médecins de l'établissement, constatant la souffrance et le stress des équipes soignantes, ont alerté la direction de l'institution. Les critères d'alerte, à prendre en considération durant les interventions, ont été une intensification de la charge de travail, la peur de la contamination, la montée en compétences rapide et la confrontation répétée à la mort. En réponse à cela, l'aide de la branche santé mentale du groupe Ramsay Santé a été sollicitée.

\section{Analyse des besoins}

À la lumière des éléments présentés précédemment, des échanges avec la direction des ressources humaines, les médecins et les responsables d'unité de soin ont permis d'identifier les réflexions suivantes, qualifiant les interventions, qui seront développées plus loin :

\section{Prise en charge groupale des équipes}

Le modèle de prise en charge s'est appuyé sur les outils de Thérapie d'Acceptation de d'Engagement (ACT). Un choix de prise en charge groupale a été fait, permettant d'accueillir au mieux la réalité collective des équipes, avec quelques occurrences d'entretiens individuels. Les outils utilisés permettent une validation émotionnelle, en première intention.

Par la suite, les intervenants guident les participant.es vers le renforcement des ressources personnelles et collectives. Il est associé à un travail sur les valeurs de chacun.e. Cela permet de concrétiser ce qui fait sens pour soi, au niveau individuel, et, collectivement, de prendre conscience des valeurs partagées dans la période douloureuse.

\section{Rythme et format des séances}

En réponse à la rigidité présentée, assurant un espace de sécurité psychique dans un premier temps, il a été primordial de proposer des séances flexibles et adaptées aux besoins des équipes. Les responsables des unités de soin ont été des liens précieux, en établissant une première alliance.

Ainsi, les temps d'échange ont été de 30 minutes à 1 heure 30 , pour des groupes de 3 à 10 personnes, avec un temps de débriefing entre les intervenant.es, à l'oral et à l'écrit de 30 
minutes à 1 heure, selon les séances. Ces temps étaient situés sur les horaires de travail des équipes et dans les unités.

Par ailleurs, les trois services n'ont pas bénéficié du même nombre de séance, en raison d'une mauvaise évaluation des besoins et d'une réévaluation n'enveloppant pas une prise en charge groupale. Sur le mois qu'a duré l'intervention, 3 à 9 groupes ont eu lieu, selon les équipes et leur disponibilité.

\section{Lieu d'intervention}

$\mathrm{Au}$ regard des conditions matérielles et du caractère urgent, il est apparu que la prise en charge devait s'effectuer à distance. Une intervention sur place aurait mobilisé un ensemble de ressources supplémentaires, aurait ajouté des facteurs de stress, à la fois pour les équipes et pour les intervenant.es, et aurait exposé l'ensemble des personnes au risque de contamination.

Pour ces raisons, les groupes se sont déroulés dans les unités, à travers un logiciel de téléconsultation.

\section{Qualité et compétences des intervenants}

Afin de faciliter l'alliance thérapeutique et d'engager la prise en charge des équipes, un binôme constitué d'infirmi.er.ère psychothérapeute et d'un.e psychologue ou psychiatre, spécialis.ée.s, par leurs expériences, dans le soin aux soignants et formé.e.s à la thérapie ACT.

La présence, dans le binôme, d'une personne ayant une expérience de terrain, dans des lieux de soins similaires aux lieux d'intervention, souvent un.e infirmi.er.ère, a favorisé la mise en lumière d'une humanité commune et le développement d'une capacité de « coping » (Lazarus et Folkman, 1984), que l'on pourrait traduire par « une adaptation psychologique au stress ».

Cette composante éclaire, à travers le dévoilement de soi, le fait qu'il a existé des occurrences similaires des éléments apportés par les équipes, et qu'il a été possible, dans ces cas précédents, de « faire avec » l'inconfort et la souffrance, puis de construire une nouvelle manière d'agir, répondant mieux aux contraintes et aux exigences des situations.

Ce partage atténue le sentiment d'isolement ressenti par certaines équipes et à différents niveaux pour la personne. Cet isolement s'exprime tant pour une personne, pour une équipe ou que pour une institution dans sa globalité. Les niveaux se traduisent sur un spectre allant de l'individu dans son quotidien, dans son équipe et dans son institution d'une part, à une équipe envers une personne seule, dans sa relation à l'institution ou dans le quotidien.

\section{Retour sur les interventions}

Les interventions ont été réalisées auprès de trois services : 1) Service Médecine Covid ; 2) Service Réanimation Covid et 3) Service Urgences Covid.

Au service Médecine Covid, huit groupes ont eu lieu. L'ensemble du personnel infirmier et aide-soignant de l'équipe de jour a participé, ainsi que la.le responsable d'Unité de Soin, la.le médecin et la.le assistant.e social.e. Les ateliers se sont déroulés dans le service, toujours en groupes, sur une durée d'un mois.

Au service Réanimation Covid, neuf groupes ont eu lieu. Le personnel soignant concerné a été le suivant : infirmi.er.ère diplômé.e d'état, aide-soignant.e, médecin, responsable d'unité de soin. Chaque personne participait lorsqu'elle le souhaitait. Les prises en charge ont eu lieu dans le service, d'abord quotidiennement, puis trois fois par semaine pendant un mois. Elles ont été en groupe ou individuelle.

Enfin, au service Urgences Covid, faisant face à une souffrance accrue des soignant.es, la direction de l'établissement a obligé cette équipe à venir au groupe d'écoute. L'alliance n'a 
pas pu être constituée en raison d'une absence de choix : le sens de l'intervention n'a pas été expliqué. Sur les trois groupes proposés, à des horaires répondant systématiquement aux contraintes du service, un seul s'est tenu concrètement. L'équipe n'a pas adhéré au dispositif.

\section{Bilan des interventions}

Nous avons fait un bilan oral à un mois après le dernier temps d'écoute, avec les responsables d'unités de soins (cadres RUS). D'autres dispositifs avaient été proposés aux équipes : sophrologie, ostéopathie, accueil par Réhalto (organisme qui propose un accompagnement psychologique pour les salariés, par téléphone ou en face à face avec un.e psychologue). Le bilan met en avant que la prise en charge groupale est la plus satisfaisante pour les équipes. Le bilan est positif pour les participant.es.

À la lumière du bilan, différents éléments positifs ont été mis en évidence :

- Le déroulement par logiciel de visioconférence, dans les services

- L'adaptabilité des horaires du groupe en fonction de la charge de travail des équipes

- La participation libre au groupe

- La prise en charge par une équipe extérieure à l'établissement

- La mise en place sous $48 \mathrm{~h}$ du dispositif

- La prise en charge par un binôme infirmier.ère diplômé.e d'état (IDE)/Psychologue, IDE/Médecin ou Médecin/Psychologue

Cependant, nous pouvons souligner un point faible, à savoir que la forte incitation de participation, par la direction, sans explication du dispositif en amont, a expliqué la difficulté de mise en place du dispositif pour le service Urgences Covid.

Trois axes d'amélioration ont été retenus :

- Proposition d'étendre la prise en charge aux équipes de nuit

- Mise en place d'un suivi individuel avec les agents qui le souhaitent à la fin du dispositif

- Proposition d'échelles d'évaluation dès le début de la prise en charge qui permettront de mesurer les variations de chaque individu

\section{La spécificité de la prise en charge}

Comme évoqué précédemment, le modèle de la prise en charge a été celui de la thérapie $\mathrm{ACT}$, et a dû prendre en considération les besoins des différents groupes.

Dans ce modèle, le but est de passer d'une rigidité psychologique à une flexibilité psychologique en intervenant de manière conjuguée sur six éléments, que nous détaillerons plus loin :

- Passé/futur : il s'agira de se centrer sur le moment présent

- Flou de qui est important pour soi : une définition précise des éléments qui sont important pour soi

- Inaction : un ensemble d'actions engagées est à envisager « avec courage »

- «Soi comme contenu» : le soi sera à observer comme un contexte

- Fusion avec les pensées : avec divers procédés, une distance pourra être prise par rapport à ses pensées

- Évitement expérientiel: les ressentis seront accueillis même s'ils sont inconfortables 
En fil rouge de cela se trouve la verbalisation et le débriefing (voir section 6), qui articulent la prise en charge.

Avant tout travail de fond, il convient de permettre de donner aux personnes les outils d'identifier l'existence d'un ressenti inconfortable. Ceux-ci, en première instance, permettent une validation émotionnelle.

La psychoéducation sur la place et le rôle des émotions dans la psyché humaine sert de socle sur lequel la personne expérimente une évaluation de celles-ci, guidée dans un premier temps, seule par la suite.

Par la suite, lorsque les individus témoignent de leur apaisement, une disponibilité d'écoute par rapport au changement apparait. L'idée n'est pas de stopper une action, mais plutôt de faire autrement, avec les ressources disponibles. Aussi, les thèmes des échanges ne se centrent plus autour des manques et failles de l'institution, insolubles, révélés par le contexte de crise sanitaire et le caractère urgent. Ils dérivent doucement vers les actions menées au quotidien en réponse à l'imprévisibilité. Celles-ci semblent se confronter à une rigidité ressentie, de l'institution vers les équipes.

Après plusieurs séances, les équipes peuvent retrouver une souplesse psychique et comportementale leur permettant de retrouver un sens dans leur pratique, au niveau individuel et collectif.

Chaque personne reconnaissant ce dont elle avait besoin pour se sentir en sécurité, ayant observé ses ressources internes et externes, il s'est agi d'explorer ce qui était important pour elles et d'envisager une gradation d'actions menant à la réalisation d'un objectif.

Métaphoriquement, cette échelle se gravit barreau après barreau. Si la personne vise un barreau trop haut pour elle, elle se retrouvera en difficulté, voire en incapacité d'agir. Aussi, ces explorations sont plus un guide vers un idéal qu'un simple objectif à atteindre. Chaque action est menée « avec courage » et engagement.

Une image pour illustrer cet engagement est celle d'un vélo. Sans élan, un vélo tombe.

Aussi, le premier coup de pédale engageant un mouvement, il n'est pas simple de le donner. La selle représente ce sur quoi la personne se repose pour poursuivre son mouvement. Ce sont les ressources internes. Le guidon, lui, sert à se diriger. C'est un guide. La roue arrière représente les ressources externes : qui solliciter en cas de difficulté. Le garde-boue et les freins représentent ce dont la personne a besoin pour se sentir en sécurité. Enfin, pour se diriger, il convient de porter son regard vers l'avant : cette orientation permet d'aller vers ce qui est important pour soi.

Ainsi, ces éléments aboutissent à une philosophie de vie. Tout au long de la vie, nos besoins changent. Ce qui est important pour nous aussi, car cela est influencé par nos interactions avec le monde extérieur, que nous ne pouvons pas contrôler. Lorsqu'une situation devient inconfortable, l'observation de nos besoins et de nos ressources permettent, tout en accueillant un ressenti inconfortable, de faire autrement, pour que la situation soit moins inconfortable, puisqu'il n'est pas possible, non plus, d'éteindre, de faire taire notre vie psychique. Tout en résonnant en nous, c'est avec courage que nous pouvons mener notre existence.

Enfin, durant la prise en charge des équipes, il nous a été témoigné régulièrement, sous différentes formes, une sorte d'impossibilité à faire, générée par un " arrêt de la pensée ».

Face à une situation créant un stress, qu'elle soit externe - rencontre avec un prédateur - ou interne - anticipation d'un entretien d'embauche -, le cerveau applique trois stratégies mettant le corps en tension : 
- L'attaque

- La sidération

- La fuite

Dans le cas d'une rencontre avec un prédateur, la logique de ces points s'élabore simplement : il s'agit de défendre le sujet avec les moyens présents en préparant le corps d'un point de vue physiologique.

Lors d'une situation plus interne, la cause du stress n'est pas clairement identifiée. Les stratégies prennent une forme différente :

- L'autocritique pour l'attaque

- La rumination pour la sidération

- L'isolement pour la fuite

Le corps réagit de la même manière face au stress, qu'il soit externe ou interne. Il prépare l'individu physiologiquement.

En outre, cet arrêt de la pensée semble représenter un état de sidération. Le sujet tourne en boucle des solutions sans finalité précise et il en vient à se sentir démuni. Par la parole et l'élaboration collective, étayées par le binôme d'intervenant.es, l'individu comme le collectif vont être amené à trouver une autre manière de faire.

Du point de vue du modèle utilisé, il s'agira pour ces ensembles de trouver de nouvelles choses importantes vers lesquelles s'orienter puis se diriger, tout en reconnaissant celles existantes. Par exemple, cela peut aboutir à la rédaction d'un projet de soin pour l'établissement.

\section{La création du sens}

Deux espaces spécifiques semblent avoir été dégagés. D’un côté, la verbalisation engage les équipes vers le binôme, puis du binôme vers les équipes, dans un mouvement se poursuivant jusqu'à l'apaisement des équipes. De l'autre, le débriefing concerne le binôme et ses partenaires. Il est le liant consolidant les séances dans le temps.

\subsection{La verbalisation}

La verbalisation d'une situation autorise une prise de recul d'un point de vue cognitif. Conjointement, en abordant le côté émotionnel, à travers le ressenti des personnes, une action d'ancrage du sujet dans le "soi contexte » plutôt que le "soi contenu » favorise une déculpabilisation des sujets.

\subsubsection{Culpabilité et abnégation}

Le sentiment de culpabilité intervient systématiquement dans les retours d'équipe. Il est en lien avec une sensation d'impuissance : peu importe les moyens utilisés, les patients sont touchés par le virus Covid. Aussi, un discours autour d'une sorte de " don de soi » enchaîne la personne à une réalité fantasmée.

Par ailleurs, il a été constaté un champ lexical de l'usure et de l'épuisement - «Je n'en peux plus », "C'est épuisant », "J'ai du mal à me concentrer », "Je suis à bout », "Je n'ai plus la force », "C'est trop pour moi», «Je ne me sens plus capable ». Face à la contrainte du travail, chaque personne réagit selon des représentations internes, qui s'élaborent au cours de la vie et à travers le contact avec des pairs, et s'expriment différemment tout au long de la 
vie. En outre, dans la population soignante, il se développe une capacité à « s'oublier» au profit de l'autre. Ce schéma, qui correspond à une représentation mentale d'abnégation, se définit par son usage courant à travers trois composantes qui se mêlent :

- Le renoncement ou sacrifice consenti pour des motifs de perfection morale et spirituelle

- Une vertu qui recouvre à la fois des qualités (bonté, modestie) et des défauts (orgueil, égoïsme)

- Le dévouement, dans sa composante active et efficace

De l'aspect positif, il s'agit d'un véritable atout, pour un.e soignant.e, que de faire preuve d'abnégation, en cela qu'iel s'efface pour se tenir au plus proche de l'autre, peu importe la tâche. C'est aussi un facteur de vulnérabilité, lorsque ce qui le soutient n'est plus présent l'usure des mécanismes compensatoires - et que le corps lâche - l'épuisement.

Le personnel soignant va par exemple :

- Revenir sur son lieu de travail pendant ses congés : «Les autres travaillent et c'est difficile, je vais les aider. "

- Renoncer à prendre des vacances : «Je ne peux pas partir en congés et abandonner les patient.es ou les collègues. »

- Faire une journée sans pause : changer de tenue à chaque changement de lieu est très contraignant, il convient de les limiter

En venant verbaliser ces actions, une autre manière de faire peut être abordée, qui prenne en compte les besoins de la personne qui l'évoque plutôt que de les mettre de côtés. Cette étape de reconnaissance des besoins semble primordiale dans l'ancrage de l'alliance et la poursuite de la prise en charge.

\subsubsection{Mise à distance du vécu par des processus langagiers}

Généralement, lorsqu'une réalité est douloureuse, le premier réflexe est de la mettre loin de soi, et non de l'accueillir. Ce point transparaît chez les soignant.es grâce à divers procédés. L'objectif principal est de protéger l'individu.

L'utilisation d'un langage technique vient brouiller les repères pour un.e non-initié.e. C'est une façon de montrer, aussi, son appartenance à un groupe particulier. Cet aspect est mitigé par la présence d'un.e infimier.ère dans le binôme : le vocabulaire technique repris, sans avoir besoin de l'expliciter pour l'autre membre du binôme, qui pourrait ne pas connaître un terme. Ainsi, la différence s'estompe entre l'équipe prise en charge et le binôme d'intervenant.es et un sentiment de proximité s'établit.

Par ailleurs, l'utilisation de l'humour permet, dans une certaine mesure, de mettre à distance des ressentis et des situations difficiles, lourdes, voire insupportables. Sur ce point, il s'avère plus complexe de faire connivence. En raison de la précision de la situation rendue comique, le propos a de grande chance d'être trop en décalage avec la réalité vécue, et il semble que l'intensité d'une situation vienne majorer cet écart.

Dans un registre proche de l'humour, et combinant en partie le vocabulaire technique, un champ lexical allégorique est parfois employé. Par exemple, au lieu de parler d'une personne en soin, le numéro de chambre est utilisé - «La 103 a encore appelé » -, ou encore la pathologie - «Le poumon de l'aile gauche a été bien pris en charge ». À nouveau, cette utilisation n'est pas malveillante. Elle relève à la fois d'une systématisation des soins, engageant une accélération des procédés classiques et d'une mesure de protection. Le repérage ne s'opère plus au niveau humain, et donc émotionnel, par identification, mais dans un espace défini dans l'établissement. 
Cela permet de poser une limite interne dans laquelle les soignant.es se préparent à intervenir. Toutefois, l'évitement émotionnel, bien qu'il soit efficace sur le court terme, perd sa force sur le long terme. La personne est tellement détachée de son vécu émotionnel que les outils de communication empathique en pâtissent.

C'est pourquoi, lors des échanges, le repérage des processus utilisés, en comparaison de ce qui était fait avant par le recours à la mémoire collective, encourage une humanité commune avec le binôme, qui favorise ce rapprochement grâce, notamment, au positionnement commun dans la thérapie ACT.

\subsection{Le débriefing}

Cette humanité commune est envisagée à travers le débriefing des différentes séances. Les binômes ont rendu compte des éléments à travers un échange oral, entre eux et avec le reste de l'équipe, et une retranscription synthétique à l'écrit. Différents temps sont à différencier dans ces étapes :

- Juste après la fin de la visio

- Après le premier échange

- La réalisation d'un écrit

- La transmission aux personnes présentes le jour même

- L'extension de ce contenu aux autres membres de l'équipe

À chaque étape, un niveau de recul est pris, permettant d'agencer les éléments de réalité (le discours des équipes) avec des notions théoriques et les réflexions des autres binômes. Cela permet, dans une certaine mesure, d'accueillir les prochains échanges.

Le premier échange est brut, souvent chargé en émotions. Le binôme vient témoigner de son ressenti vis-à-vis de l'ambiance de l'entretien. Cette identification réalisée, il convient de la valider par des éléments concrets et/ou propres aux personnes intervenantes.

Cet échange brut fait, la réflexion peut prendre sa place : c'est le premier maillon qui est posé - soit vers la théorie, soit vers les anciennes séances.

La conceptualisation se poursuit par l'écrit. Elle permet, premièrement, de laisser une trace et, deuxièmement, de définir le champ de la réflexion. Pour rappel, celle-ci se centre principalement vers une élaboration sécurisante pour le binôme.

La quatrième étape permet de synthétiser les éléments ressentis, oraux et écrits à d'autres. Ceux-ci sont souvent questionnés et mis «en attente» jusqu'à l'extension aux autres membres.

Cette transmission, enfin, clôt la séance, plusieurs jours, souvent, après sa fin « réelle». Entre-temps, chaque personne dans le binôme a pu faire sienne la problématique du groupe.

Il est à noter que le débriefing ne respecte pas nécessairement l'ordre présenté. Ainsi, après ces moments, le binôme est prêt pour accueillir à nouveau les soignant.es.

\subsection{Continuité des séances}

En présentant une disponibilité face aux contraintes institutionnelles, le binôme assure un espace dans lequel les équipes peuvent expérimenter de nouvelles manières de faire. Il a été remarqué qu'en définissant une période d'intervention, les équipes semblaient plus volontaires. Cette partie «psychologique» est un facilitateur pour l'activation comportementale. Celle-ci se définit comme un outil visant le réengagement dans des activités potentiellement bénéfiques. Ce n'est pas parce que la méditation marche pour une personne que c'est une activité bénéfique pour une autre, mais il convient d'expérimenter. 
Un ensemble de cognitions viennent freiner, voire empêcher, la plupart du temps, le passage à l'action. La verbalisation des moyens de faire, grâce à l'humanité commune, permet aux personnes de penser l'action. Le témoignage de sa réalisation, ou de la pensée de la réalisation, crée une opportunité de faire par le soutien de deux composantes :

- Psychologique : bien-être, satisfaction, fierté, etc.

- Sociale : encouragement, humanité commune, empathie, etc.

En s'appuyant sur un modèle bio-psycho-social du trouble, la prise en charge sort d'un « tout institutionnel » ou d'un « tout individuel ». C'est un entre-deux qui se dessine à mesure des séances. Les institutions, dans leur démarche, génèrent certes des contraintes et des résistances, mais les individus aussi, de leur côté, créent les leur. Aussi, en affinant chaque domaine de l'existence, au gré des échanges, les équipes arrivent à gagner en flexibilité psychologique et comportementale.

\section{La suite de la prise en charge}

La question qui se pose après avoir accueilli plusieurs fois les équipes est la poursuite de l'activité et sous quelles formes.

En venant questionner :

- La réalité externe ;

- La réalité interne ;

- Les choses importantes pour l'équipe ;

- Les choses importantes pour les individus.

Le travail au sein de l'institution peut être amené à se transformer.

La première forme prise en charge, par ailleurs, s'efface dans le temps pour laisser place à un autre suivi. En persistant sur un premier modèle, les besoins spécifiques ne sont plus abordés, et il semble que les réflexions tendraient à se noyer dans des considérations insolubles, voire indicibles.

Face à la charge de travail, une prise en charge spécifique peut être envisagée par une ou plusieurs personnes. Ce faisant, le poste ne sera peut-être plus occupé de la même manière temps partiel ou arrêt maladie - et, donc, la prise en charge par l'équipe. Aussi, selon les politiques des établissements, nous imaginons que le temps de travail peut être laissé vacant, complété ou remplacé.

Sur ces points, les institutions ont clairement la main. Dans le code du travail, la constitution d'un syndicat autorise un lieu d'échange entre les différents corps de métier. D'un lieu à un autre, celui-là est plus ou moins présent. L'histoire de l'établissement appuie la compréhension de l'état des lieux.

Sur ces deux exemples, il est clair que l'individu, seul, semble démuni.

D'où la nécessité d'identifier les choses importantes pour soi. Du côté de l'équipe, elles peuvent se concrétiser sous la forme de la rédaction d'un projet de soin, venant soutenir une nouvelle manière de faire, et où chaque personne a la possibilité, dans l'idéal, d'exprimer son avis.

Du côté de l'individu, le questionnement des éléments importants ouvre deux éventualités : rester ou partir. En pointant l'abnégation que les soignant.es démontrent, les besoins individuels émergent. Concrètement, un investissement différent de l'extérieur pourrait autant soutenir le travail au sein de l'institution qu'encourager un départ.

Par ailleurs, concernant la temporalité de la prise en charge, une question peut se poser : au bout de combien de temps une urgence n'en est plus une? 
La vocation du soutien des équipes n'est pas de garantir la viabilité économique d'une institution, mais bel et bien de préserver la santé mentale des soignant.es, en leur donnant les moyens d'identifier les ressources dont iels ont besoin pour se sentir en sécurité. À travers l'ensemble de la prise en charge, il est régulièrement rappelé un principe de réalité : il est normal, tant au niveau biologique, psychologique et social, de ressentir de la souffrance dans une situation qui en génère.

Après une phase d'apaisement, une réflexion s'engage autour d'une mise en action, organisée autour d'éléments importants pour les personnes. C'est alors que survient la nécessité d'expérimenter. Rester dans une phase d'observation, de pondération, d'analyse et de planification est confortable pour certaines personnes. Il s'agit pourtant d'un évitement et donc d'une résistance face au changement.

En venant stopper la prise en charge «d'urgence », les personnes peuvent ainsi se saisir de leurs ressources. Si elles en ressentent le besoin, elles iront par leur propre moyen solliciter un réseau différent. Cela concerne des situations individuelles spécifiques et ne peut pas s'étendre à l'ensemble du personnel soignant.

\section{Témoignages}

Après avoir détaillé en quoi la manière dont le soutien des équipes s'est organisé d'une façon spécifique, il nous est apparu intéressant de pouvoir témoigner plus directement de l'expérience de chaque personne ayant pu intervenir auprès des équipes de MCO.

Les témoignages suivants se sont organisés autour des trois questions, traitées librement par chaque personne.

1. Quel a été votre ressenti lors de la création du dispositif d'intervention ?

2. Comment se sont déroulées les différentes séances auxquelles vous avez participé ?

3. Du point de vue soignant, que retenez-vous principalement de cette expérience ?

\section{Marie-Françoise, infirmière :}

Au début de l'aventure j'étais partagée sur ma participation entre l'envie d'apporter ma contribution dans cette crise sanitaire, à défaut d'être au front comme certains de mes collègues infirmiers, et le sentiment de ne pas être légitime. Mes collègues m'ont fortement encouragée à participer, au regard de mon expertise de terrain en milieu hospitalier, et pour mieux installer un climat de confiance avec les équipes. Le fait de savoir que j'allais intervenir avec un binôme psychologue ou psychiatre m'a mis en confiance et je me suis ainsi lancée dans l'aventure.

J'ai participé à environ 2 à 3 visio par semaine en dehors de mon temps de travail habituel. J'ai eu la chance de suivre l'équipe de Réa Covid qui a été d'emblée très participative à nos interventions. Le cadre de santé, très coopératif nous a permis de rentrer au cœur du service de Réa. Grâce à la visio, différentes équipes se sont succédé. Même si la charge de travail était lourde, chaque membre a pu se libérer pour venir échanger sur son vécu. J'ai été frappée par la cohésion des équipes, le dévouement, l'abnégation et la mise à distance des ressentis.

Je retiens la réactivité de notre service à la demande, la rapidité de mise en place du dispositif et l'adaptabilité que cela nous a demandée. Je retiens également la complémentarité du binôme infirmier/psychologue ou psychiatre. 


\section{Ivan, psychologue :}

Dans un premier temps, j'ai été témoin de la mise en place du dispositif. J'ai vu plusieurs collègues organiser au plus vite une prise en charge nouvelle. J'étais partagé entre ma disponibilité pour intervenir et mon sentiment de ne pas faire comme il faudrait pour prendre en charge, dans ce contexte d'urgence. En dialoguant avec l'équipe, j'ai pu mettre de côté mon appréhension et me concentrer sur les éléments de débriefing. Ce partage d'expérience a, je pense, facilité mon acceptation du dispositif: il ne semble certes pas parfait, sauf qu'il existe, à défaut de ne rien faire. Par ailleurs, il a été rassurant pour moi de constater que je n'allais pas intervenir seul, en amont ou en aval. J'aurais toujours le soutien d'un groupe de personnes. Par la suite, lorsqu'il m'a été proposé d'être intervenant, je me suis rendu compte que je n'avais pas une place si externe que je le pensais dans la création du dispositif.

Les différentes séances auxquelles j'ai participé étaient en dehors de mes horaires de travail. J'ai accepté cet inconfort pour répondre à un élément important pour moi en tant que soignant. Les séances auraient pu se dérouler autrement. Les équipes ne semblaient pas avoir été prévenues de notre intervention. Le premier accueil a été très chaleureux, et les suivants beaucoup plus distants : un premier dépôt de ressentis inconfortables et d'anecdotes désagréables ayant été fait, c'est comme si les équipes n'avaient pas envie d'élaborer davantage. Alors que nous avions fait preuve de flexibilité, dans les horaires notamment - je suis venu un jour en dehors de mon temps de travail habituel pour répondre à la demande de l'équipe prise en charge-, une fluidité est venue à manquer. Le dialogue ne semblait plus possible.

En tant que soignant, je retiens, dans ce contexte d'urgence, qu'il est possible d'intervenir et de prendre en charge avec des outils changeants et adaptables, et ce à plusieurs.

\section{Florent, psychologue :}

Dans ce dispositif d'intervention, j'ai constaté une difficulté de penser le soin dans l'urgence et de le faire de manière adaptée. Un vrai questionnement s'est posé autour de l'outil de visio en ce sens qu'il pouvait générer une interférence dans le travail et dans la manière dont le lien se créait. Une des craintes a été qu'il puisse dénaturer la relation ou, au contraire, créer de la méfiance ou un vécu d'intrusion chez les équipes.

A travers les séances, il est apparu que le fait d'être en binôme, en réelle collaboration, a été très pertinent par rapport au contexte d'urgence et de la présence d'un outil virtuel dans les services. A deux, il a été possible d'être au plus proche des gens accompagnés. Seul, sans l'appui d'une pluridisciplinarité dans le binôme et de la polyvalence des approches, il aurait été difficile d'intervenir.

Je retiens principalement la nécessité d'une collaboration véritable, où la pluri- et transdisciplinarité sont essentielles pour construire, établir, organiser et rendre viable un parcours de soin adapté. Aussi, la possibilité de pouvoir compter sur les nouvelles technologies pour seconder la démarche thérapeutique a été centrale à travers cette expérience. 


\section{Laurent, psychologue :}

Tout d'abord, je me suis questionné sur la pertinence d'un outil de visioconférence. Le non-verbal qui est perçu dans les échanges en face à face serait-il accessible? Est-ce que l'on pourrait voir le groupe dans sa totalité ? Par ailleurs, je me suis demandé quelle était l'origine de la demande : étaitce de l'équipe? Des cadres? Finalement, quelle(s) raison(s) venai(en)t la soutenir?

Durant cette phase préparatoire, la démarche a été grandement facilitée par la présence, avec moi, d'une IDE qui avait déjà travaillé dans un service de réanimation. Aussi, je suis allé me renseigner sur les pratiques d'autres collègues. Leurs témoignages par rapport à des catastrophes, surtout humanitaires, ont pu éclaircir d'autres manières de faire. En parallèle de cela, je ne me suis d'abord pas senti à la hauteur, dépassé par des enjeux trop importants. Après le premier groupe, j'ai constaté que la relation avec les équipes s'était facilement établie. Une difficulté a pu émerger du côté du logiciel, ainsi que la présence ou non des cadres lors des séances. Parfois, je sentais la lourdeur des enjeux institutionnels.

J'ai été surpris de constater la rapidité du dispositif. Après trois semaines, les besoins avaient changé. Je me suis aussi senti en capacité de me rendre très disponible, très vite, mais sans, toutefois, envisager à quoi cela venait répondre. À travers les séances, j'ai observé une dynamique " classique » dans le groupe. Après coup, j'ai trouvé ma position proche d'un soin de coordination.

\section{Conclusion}

Il convient de rappeler que le soutien des équipes de santé mentales aux équipes MCO s'est organisé dans un contexte d'urgence, celui de la crise Covid.

L'analyse des besoins des équipes, par l'échange avec les cadres et les médecins des différents services, a permis d'identifier trois points :

- La nécessité d'un groupe plutôt qu'un suivi individuel

- Le soutien à distance

- L'intégration de différentes professions

Les interventions se sont généralement bien déroulées. Le fil rouge de celles-ci a été la thérapie ACT, par un binôme (médical et paramédical, ou paramédical).

Le bilan des interventions a démontré que le groupe s'est vu renforcer ses ressources collectives préexistantes; que le soutien à distance permettait d'éviter d'intervenir dans des locaux à haute densité virologique; que l'intégration de différentes professions dans le binôme a facilité l'alliance avec les équipes soutenues.

La prise en charge, par l'utilisation du modèle thérapeutique de l'ACT, parcourt différentes notions dans l'élaboration. En identifiant sa situation, un individu sera en mesure d'envisager une possibilité de changement, par l'utilisation de ses ressources et, ainsi, s'engager vers le changement face à un arrêt de la pensée, caractéristique de l'état de souffrance auquel la personne est confrontée.

À travers un temps de verbalisation, des équipes vers le binôme d'intervenant.es, et celui de débriefing, entre le binôme et les autres intervenant.es, un sens cohérent s'établit. 
Par ailleurs, un suivi ne pouvant pas se constituer comme étant immuable, si la quête de l'autonomie est un des objectifs d'une prise en charge, il convient d'introduire une temporalité permettant de penser une suite, un après.

C'est cet « après » qui a longtemps fait l'objet de nombreuses questions. Encore à ce jour, l'embardée contrainte de la crise Covid marque les pratiques des soignant.es, qui ont su, à travers différents dispositifs, retrouver un sens dans leur métier.

Il s'agira d'ancrer la nécessité de prendre soin de la santé mentale dans les différents corps de métier, en établissant des partenariats durables avec l'ensemble des instances de soin. La piste associative semble être, à ce jour, prometteuse quant à cette idée.

\section{Références}

Bourgognon François, Penet Claude. (2021). La Thérapie d'acceptation et d'engagement, Paris : Que sais-je ?.

Hayes Steven. (2004). Acceptance and commitment therapy, relational frame theory, and the third wave of behavioral and cognitive therapies. Behavior Therapy, vol. $35, \mathrm{n}^{\circ} 4$, p. 639-665. https://www.sciencedirect.com/science/ article/abs/pii/S0005789404800133

Lazarus Richard, Folkman Susan. (1984). Stress, appraisal, and coping, New York: Springer.

Monestès Jean-Louis, Villatte Matthieu. (2011). La thérapie d'acceptation et d'engagement : $A C T$, Paris : Elsevier Masson.

Russ Harris. (2017). Passez à l'ACT, Louvain-la-Neuve : De Boeck Supérieur.

Schoendorff Benjamin, Grand Jana, Bolduc Marie-France. (2011). La Thérapie d'acceptation et d'engagement : Guide clinique, Louvain-la-Neuve : De Boeck Supérieur. 